\title{
CAM Cellulose microfibers (CMFs) reinforced smart self-healing polymeric composite coatings for corrosion protection of steel

Muddasir Nawaz ${ }^{1}$, Sehrish Habib ${ }^{1}$, Adnan Khan ${ }^{1}$, R.A Shakoor ${ }^{1 *}$, Ramazan Kahraman ${ }^{2}$

Faculty and Postdoc, Science and Engineering
${ }^{1}$ Center for advanced Materials (CAM), Qatar University, Doha, Qatar

2Department of Chemical Engineering, Qatar University, Doha, Qatar

\section{Research background and Motivation}

Corrosion degradation of materials and structures is one of important issues that leads to investment loss. Two main approaches, an active and passive corrosion protection. The passive corrosion protection prevent contact of the material with corrosive environment, while active corrosion protection aims to decrease the corrosion rate while main barrier is damaged.

Combination of both approaches provide the reliable and sustainable longterm corrosion protection of metallic structures which are in direct contact with corrosive environment. Nanocomposite polymeric coating with a mixture of eco-friendly Inhibitor applied on carbon steel for corrosion protection

\section{Introduction}
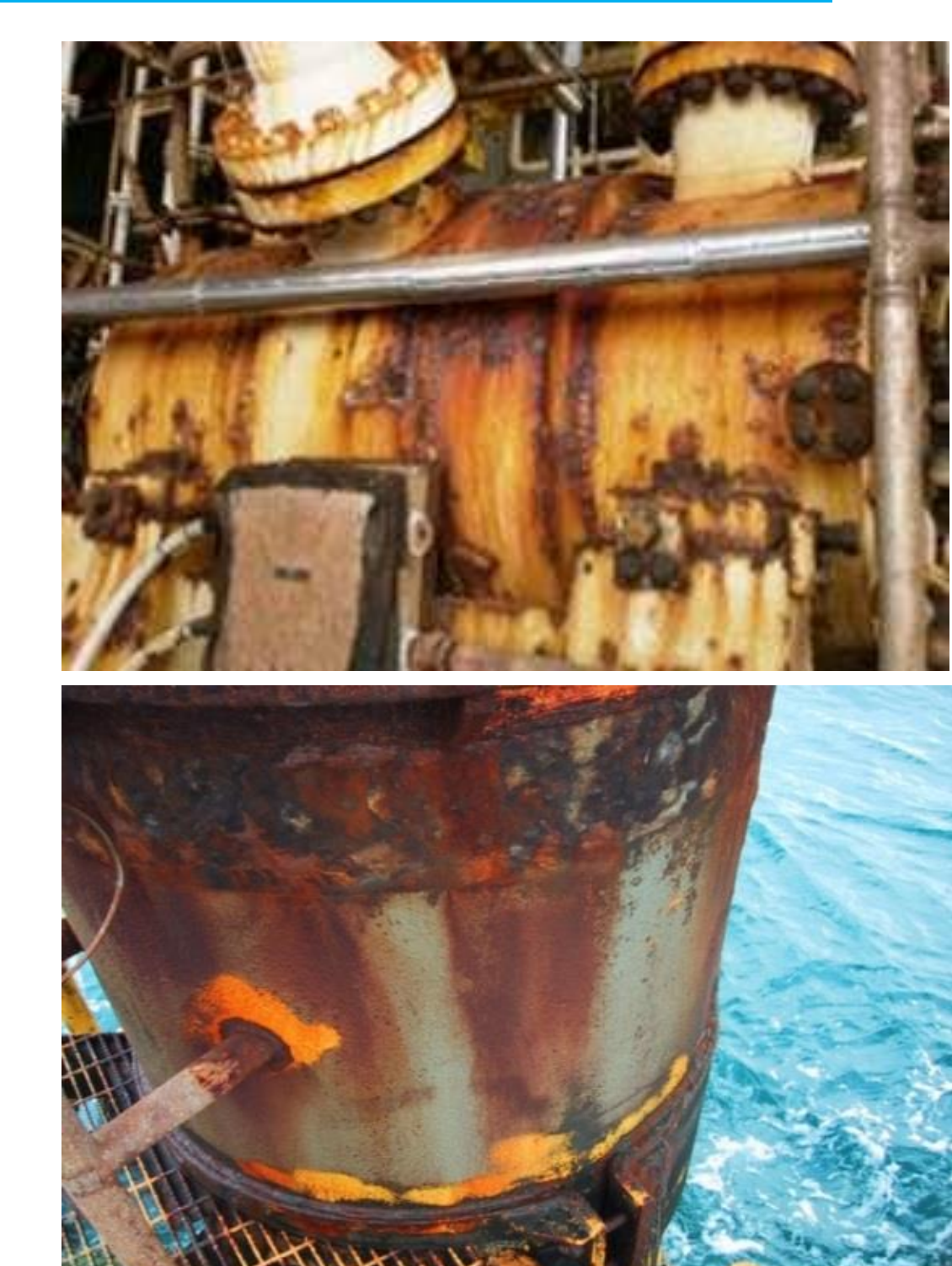

\section{Methodology}

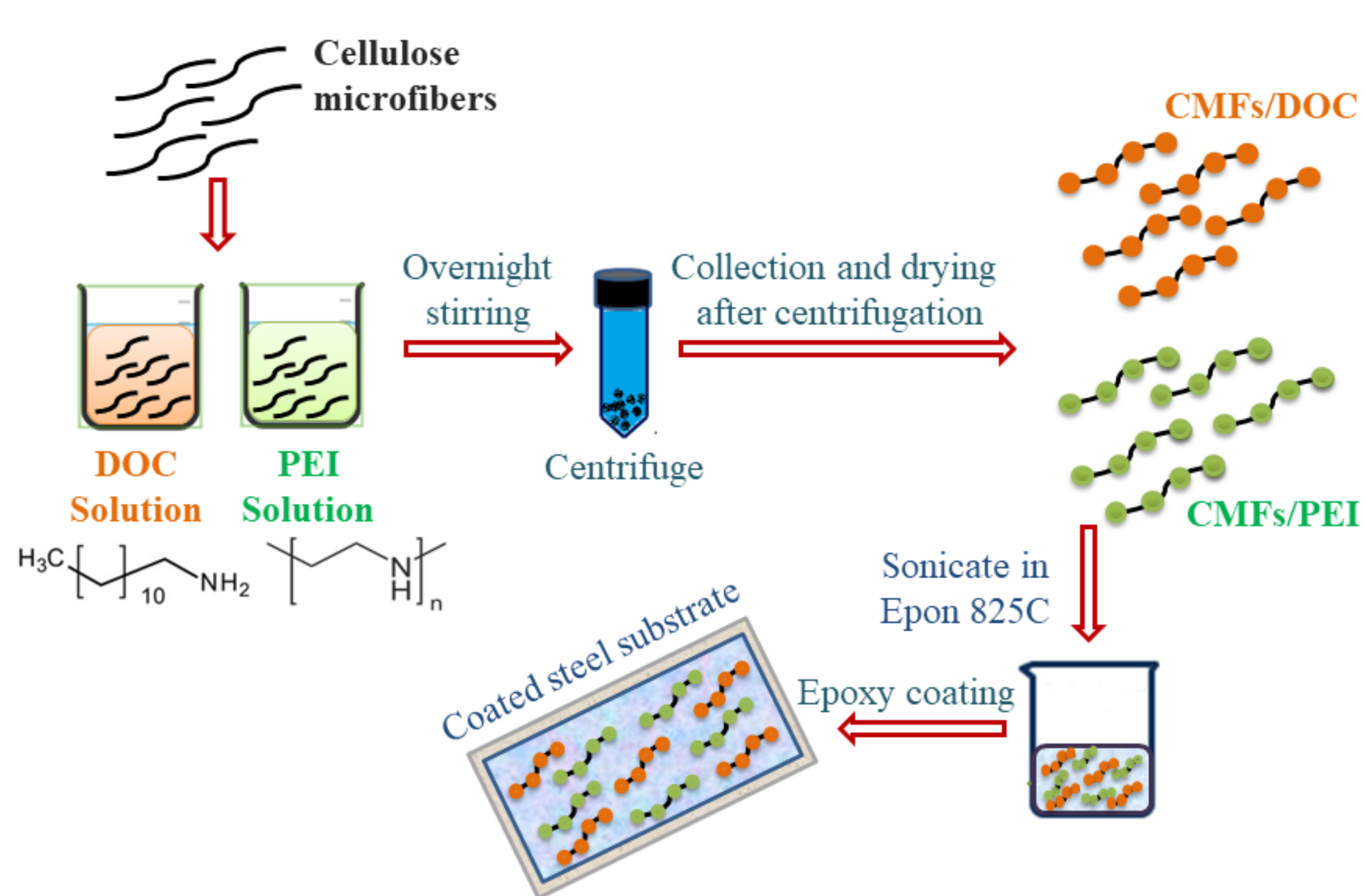

\section{Results and Discussion}

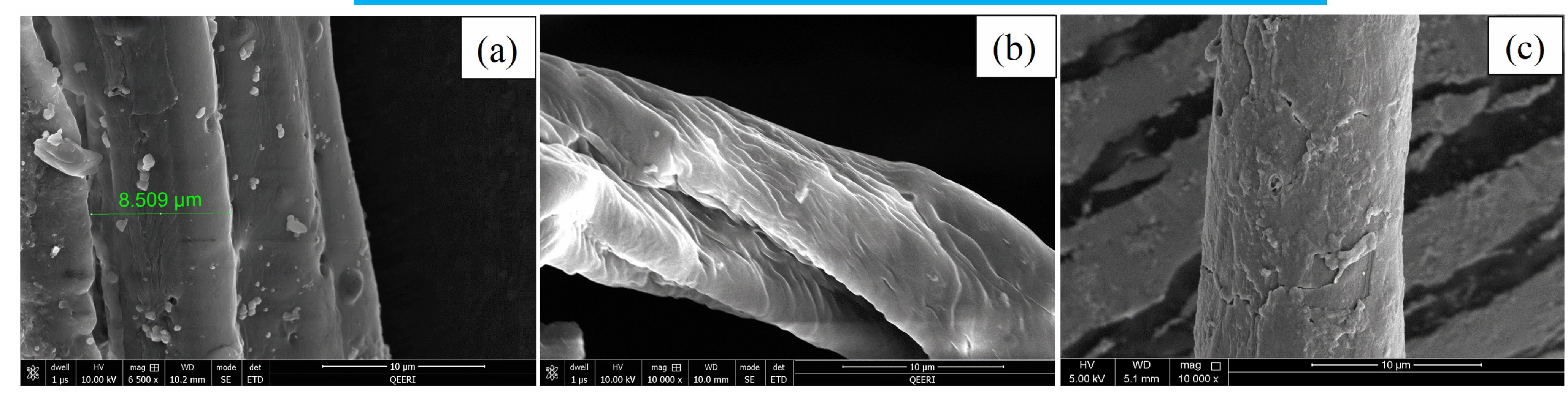

Figure 1. SEM images for (CMFs), (b) CMFs/DOC and (c) CMFs/PEI

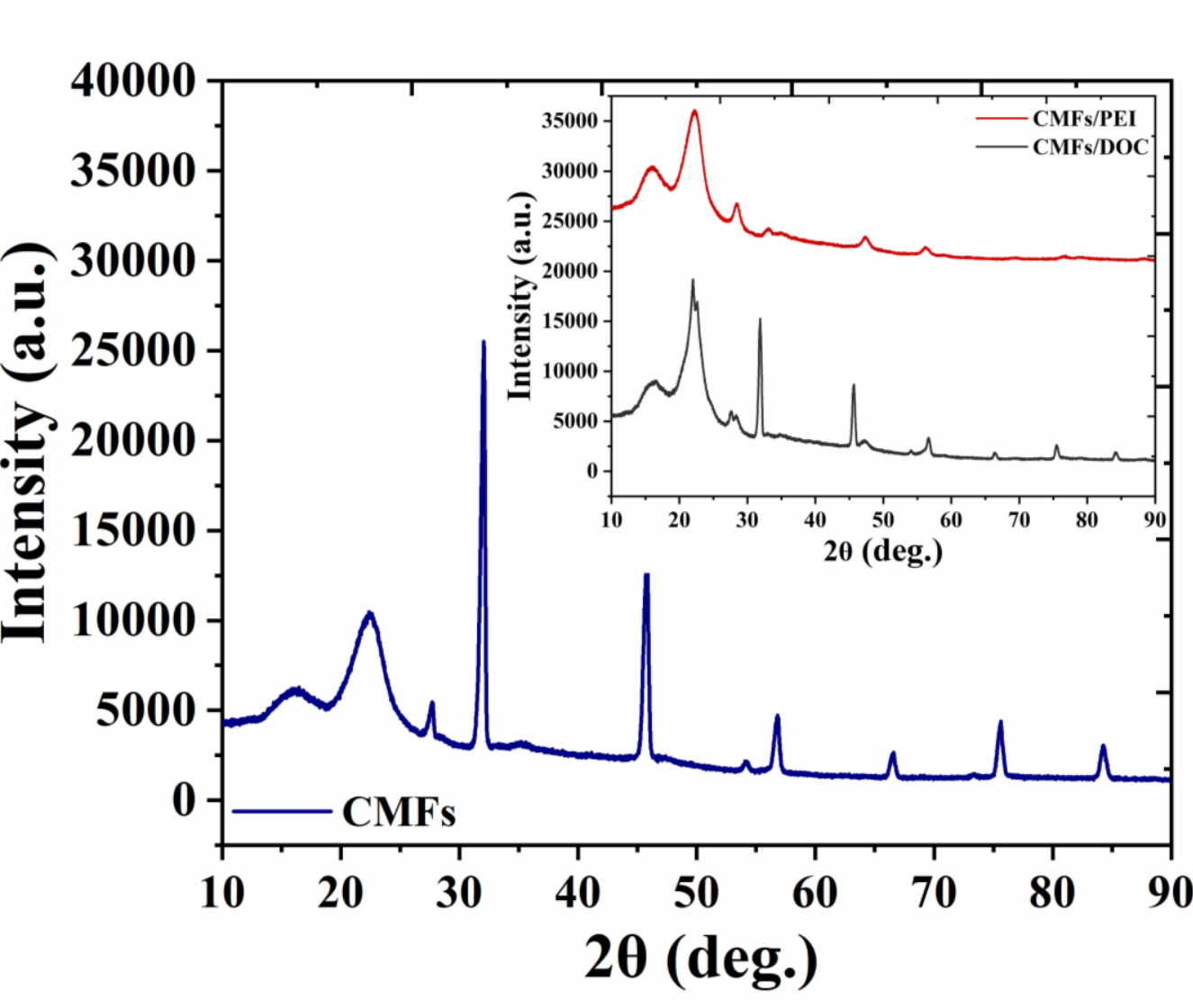

Figure 2. XRD/FTIR for CMFs, CMFs/DOC and CMFs/PEI

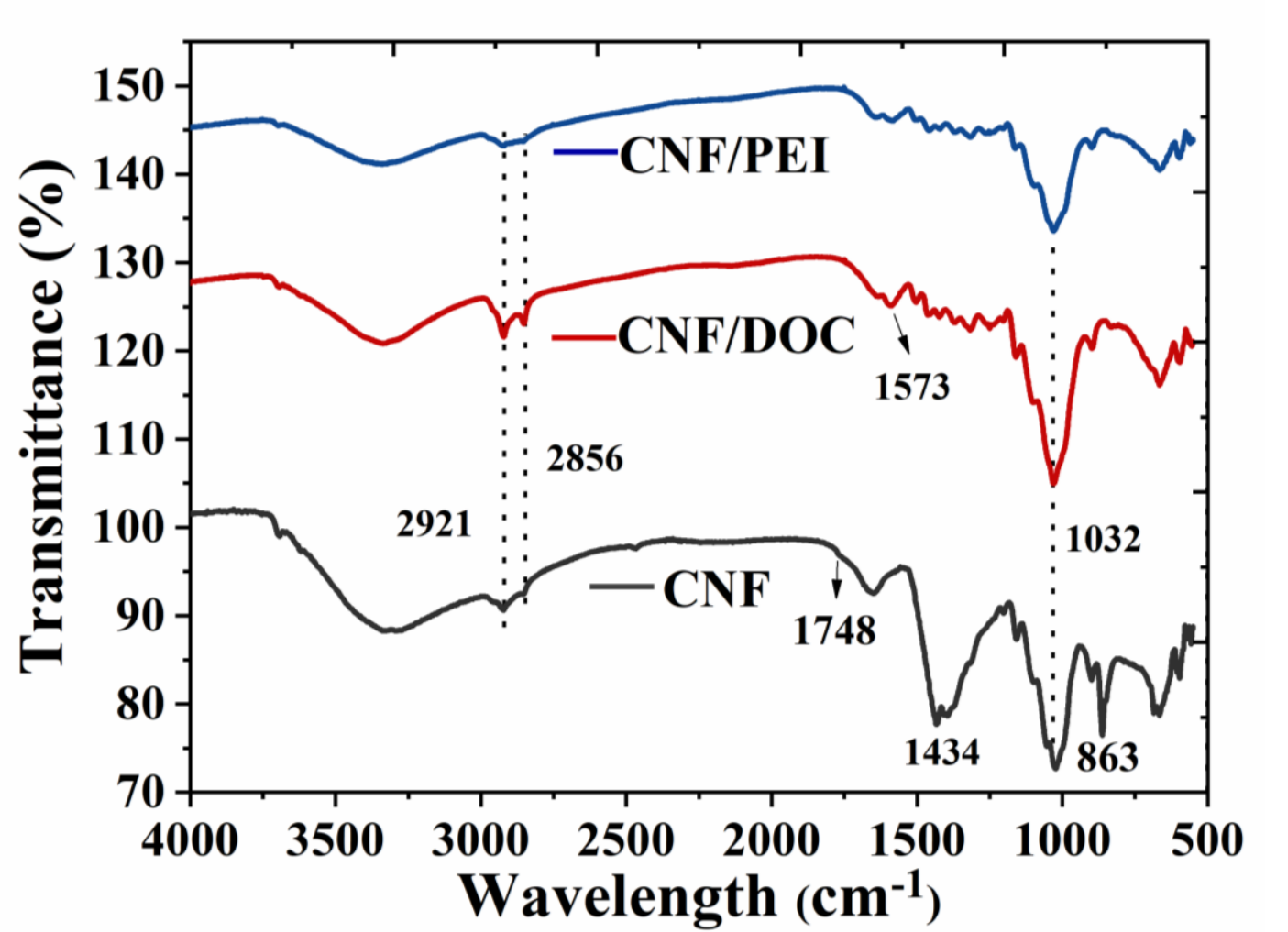

Figure 4. FTIR spectrum of CMFs, CMFs/DOC and CMFs/PEI.

Figure 5. Bode and phase angle plot for $(a, b)$ reference coating and $(c, d)$ for modified coating.

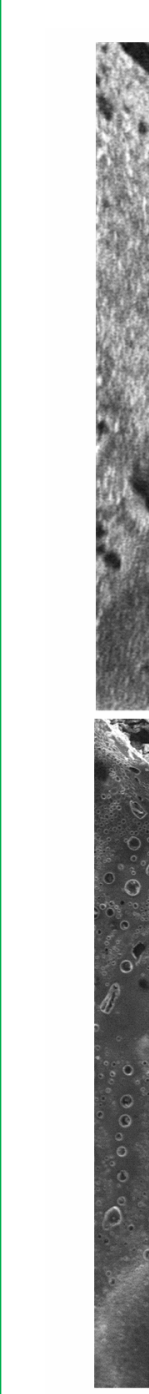
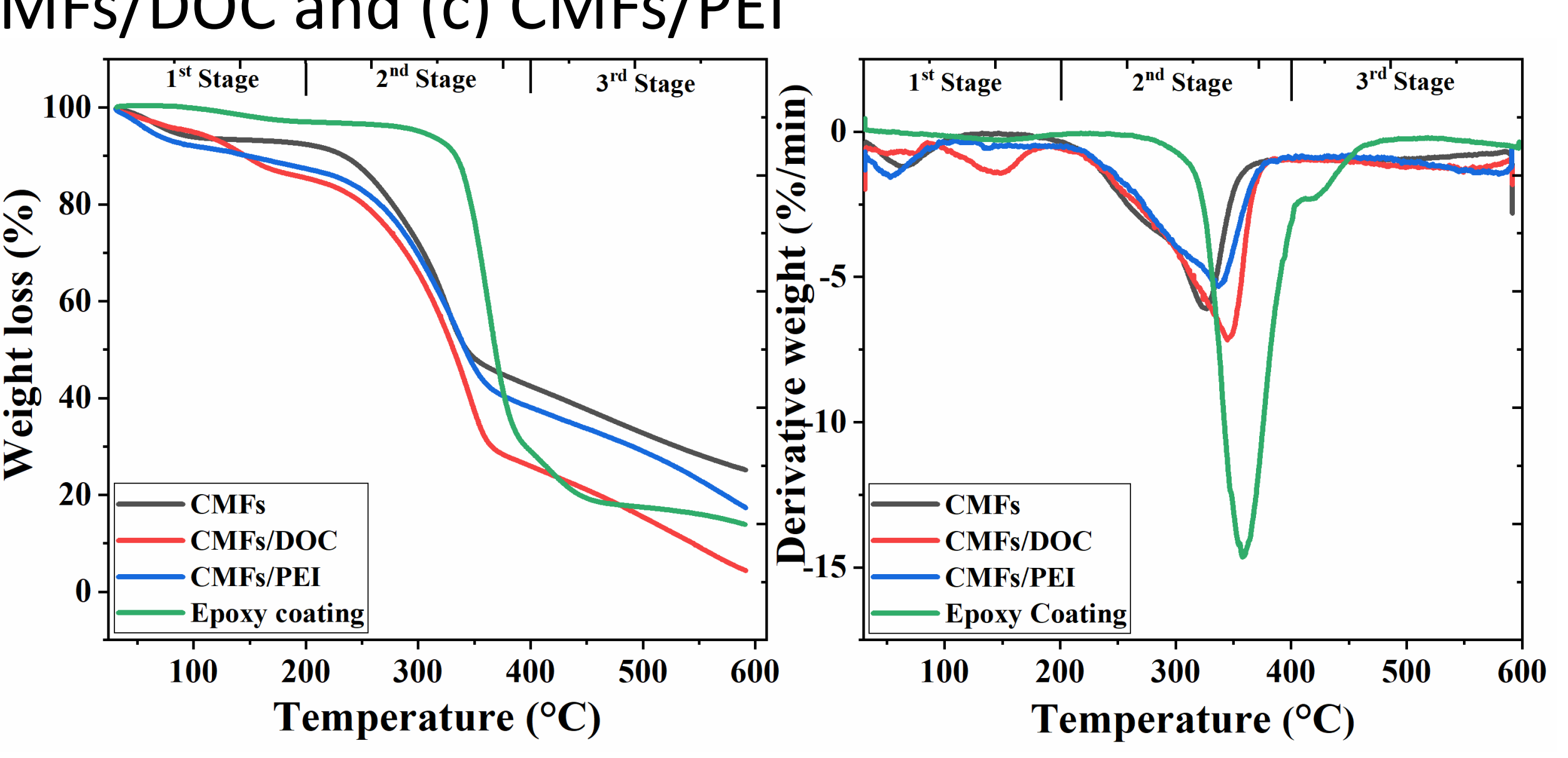

Figure 3. TGA and DTA graph for CMFs, CMFs/DOC CMFs/PEI
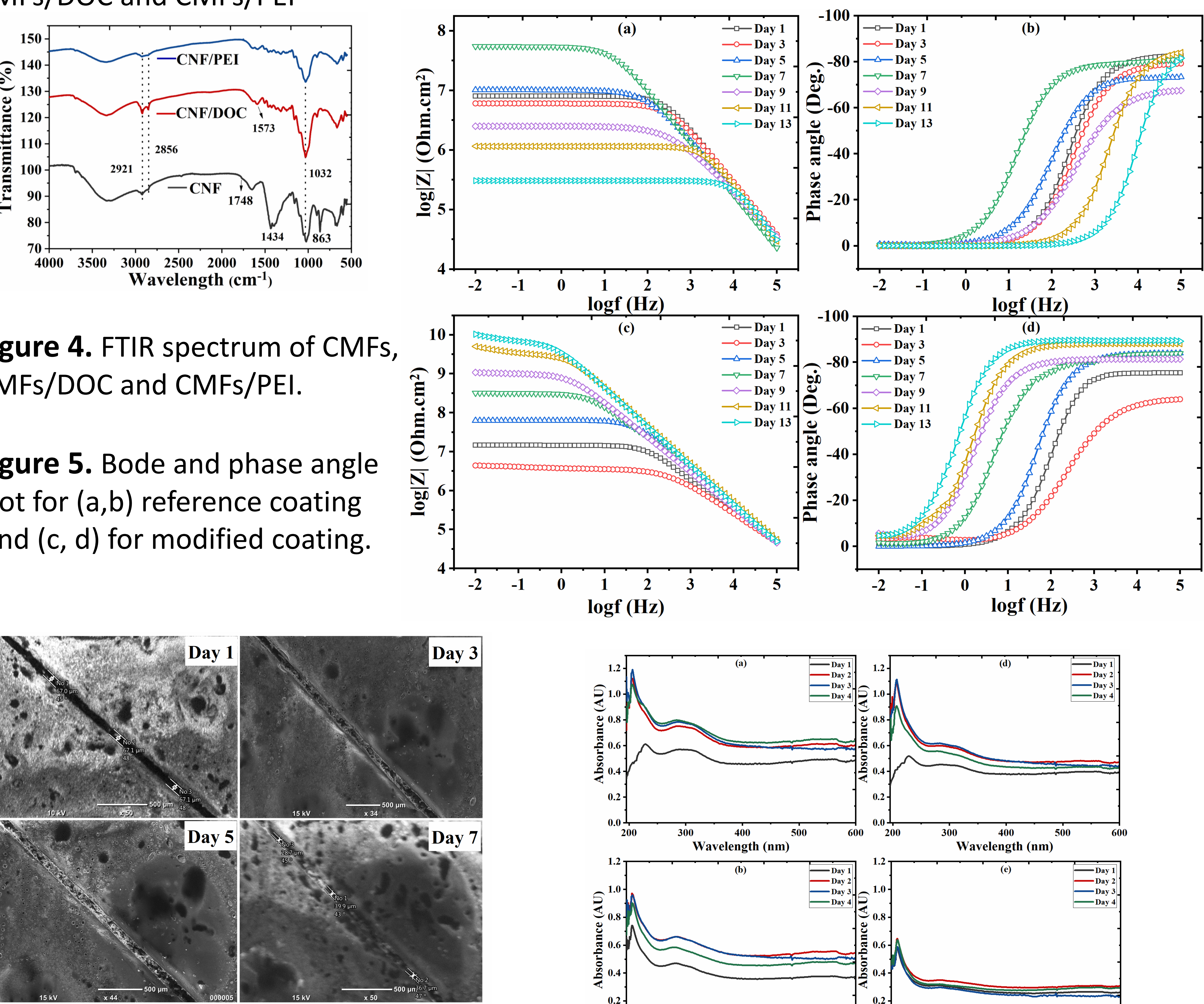

PEI

DOC

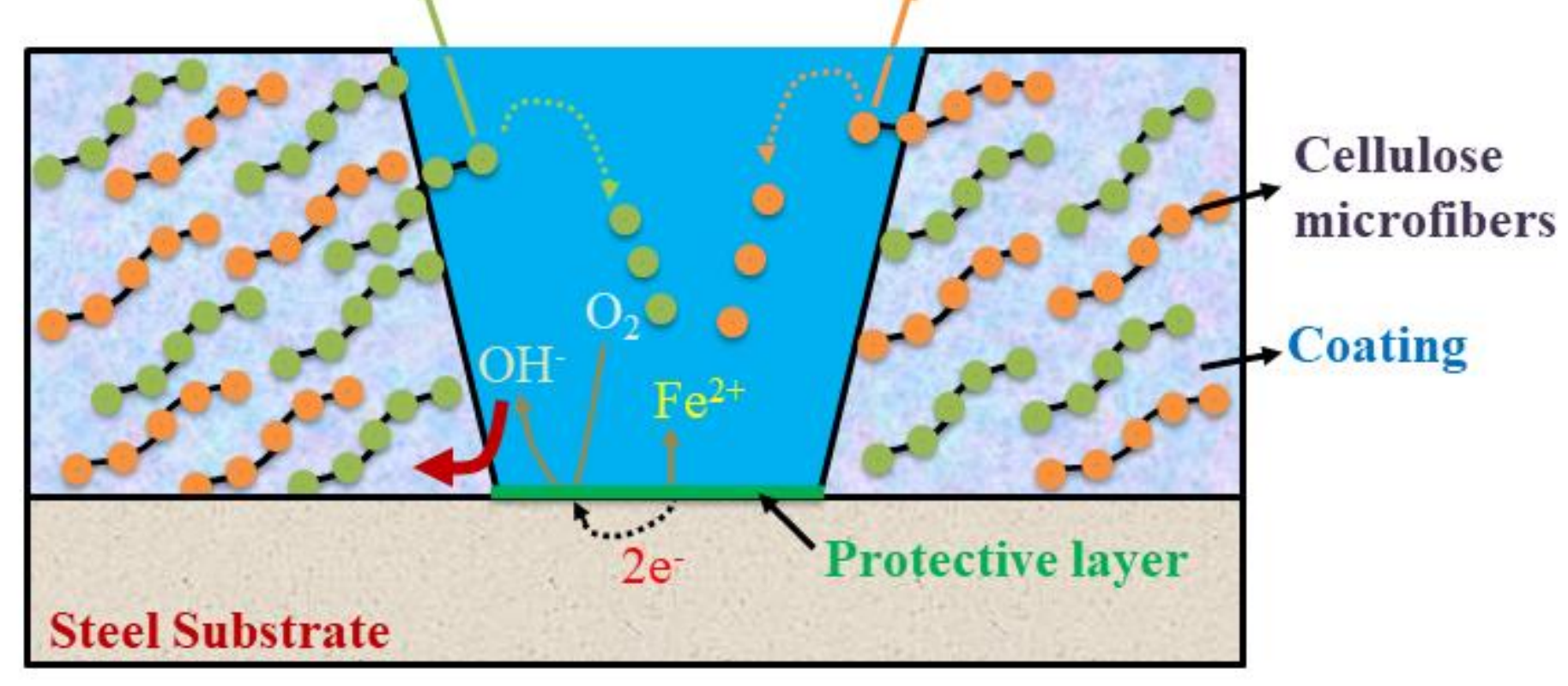

Figure 6. The SEM images of self-healing with time in modified coating.
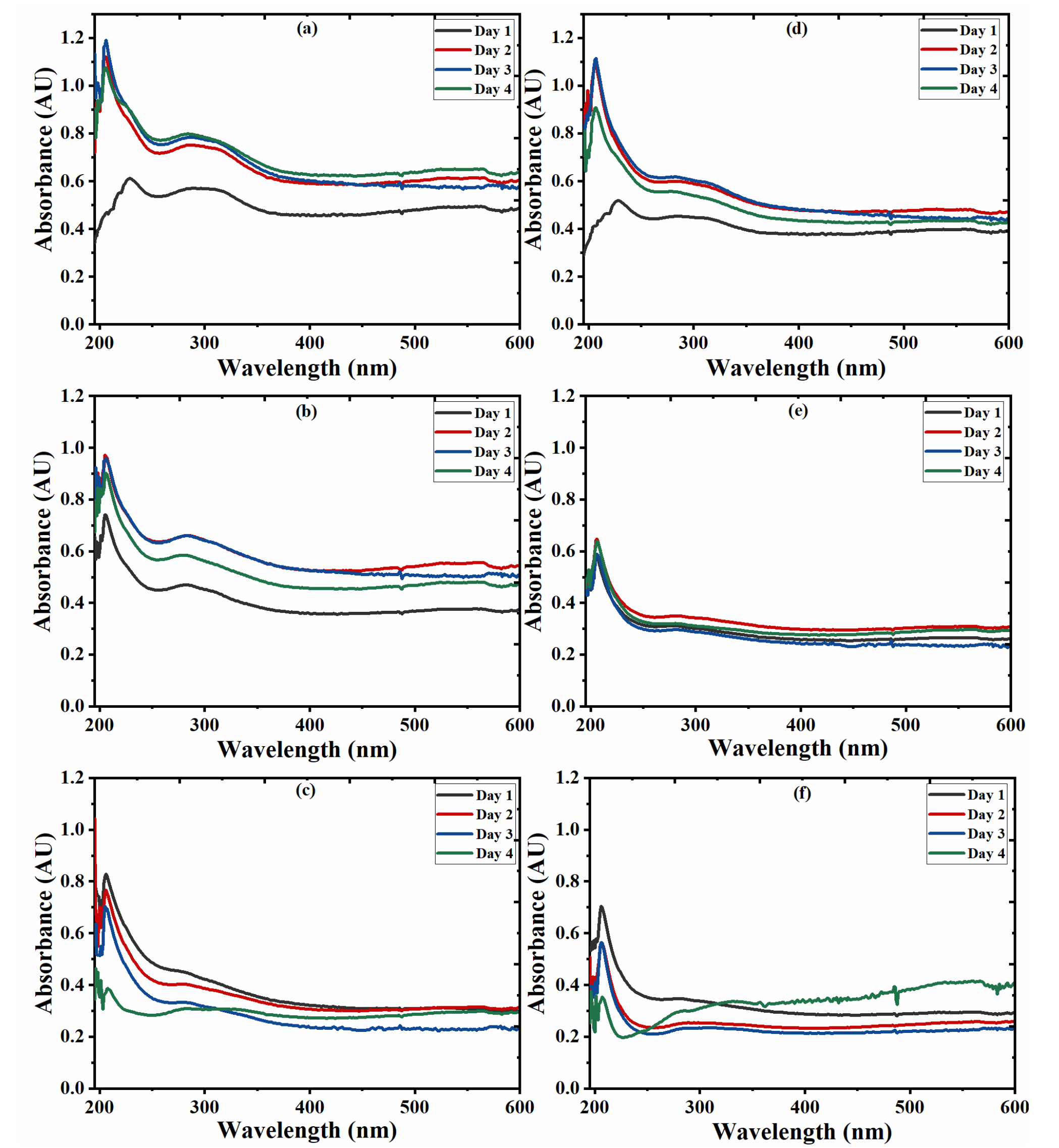

Figure 7. Uv-vis analysis for CMFs/DOC at $\mathrm{pH}$ 2,5 and 7 .

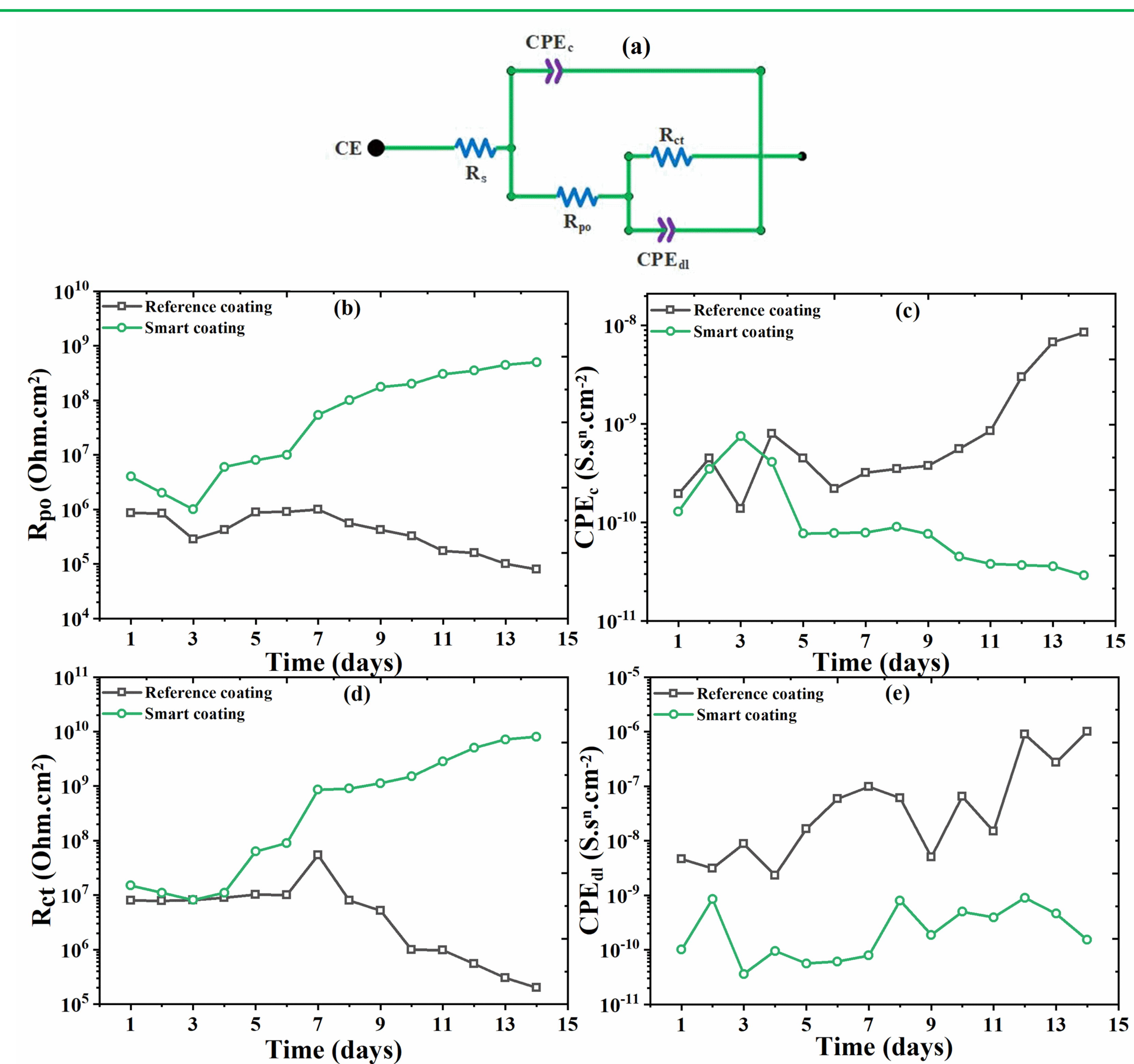

Figure 5. Bode and phase angle plot for $(a, b)$ reference coating and (c, d) for modified coating.

\section{Summery}

- Cellulose microfibers (CMFs) were synthesized and used as a carrier for loading of inhibitor (DOC) and as a self-healing agent (PEI).

- TGA and FTIR analyses confirm the loading of DOC and PEI into CMFs. UV-vis analysis indicates the time dependence and $\mathrm{pH}$ sensitive release of loaded DOC and PEI from CMFCs.

- The smart self-healing polymeric composite coatings demonstrate improved anticorrosion properties as compared to the reference coatings due to increase in charge transfer resistance $\left(R_{c t}\right)$ and pore resistance $\left(R_{p o}\right)$ with increasing immersion time and decrease in constant phase element $\left(C P E_{c}\right)$ with increasing immersion time.

- The enhanced corrosion resistance of the smart coatings can be attributed to the efficient release and adsorption of loaded DOC and PEI onto steel substrate.

\section{Acknowledgement}

This publication was made possible by NPRP Grant 11S-1226170132 from Qatar National Research Fund (a member of the Qatar Foundation). Statements made herein are solely the responsibility of the authors. 